\title{
Measuring the double-drift illusion with hand tracking
}

\author{
Bernard Marius 't Hart 1," \\ Denise Y.P. Henriques 1 \\ Patrick Cavanagh 1,2,3
}

\begin{abstract}
1: Centre for Vision Research, York University, Toronto, ON M3J1P3, Canada
2: Department of Psychology, Glendon College, Toronto, ON M4N3M6, Canada

3: Department of Psychological and Brain Sciences, Dartmouth College, Hanover, NJ 03755 , USA
\end{abstract}

\author{
*: Corresponding author: \\ Marius 't Hart \\ Centre for Vision Research \\ York University \\ Toronto, ON M3J1P3 \\ Canada \\ thartbm@gmail.com
}

\section{Abstract}

If a gabor pattern drifts in one direction while its internal texture drifts in the orthogonal direction, its perceived position deviates further and further away from its true path. We first evaluated the illusion using manual tracking. Participants followed the gabor with a stylus on a drawing tablet that coincided optically with the horizontal monitor surface. Their hand and the stylus were not visible during the tracking. The magnitude of the tracking illusion corresponded closely to previous perceptual and pointing measures indicating that manual tracking is a valid measure for the illusion. This allowed us to use it in a second experiment to capture the behavior of the illusion as it eventually degrades and breaks down in single trials. Specifically, the deviation of the gabor stops accumulating at some point and either stays at a fixed offset or resets toward the veridical position. To report the perceived trajectory of the gabor, participants drew it after the gabor was removed from the monitor. Resets were detected and analyzed and they suggest that there is a spatial limit beyond which the illusion saturates or resets.

\section{Introduction}

Encoding the position of objects in the world is necessary for the myriad of visually guided motor tasks that we do every day. It is no surprise then that the brain has several mechanisms to gauge and update the position of objects in the world. For example, when a target is moving, its motion can influence its perceived location, making it appear to be slightly ahead of its true location, e.g. the flash lag (Nijhawan, 1994); or the flash grab effect 
(Cavanagh $\&$ Anstis, 2013). These cases of position extrapolation may be functional, helping the motor system overcome inevitable neural delays in targeting a moving object (Duhamel et al., 1992; Hogendoorn, 2020; Nijhawan, 1994). A stronger and very different motioninduced position shift arises when a moving gabor has internal motion orthogonal to its path and is viewed in the periphery (Gurnsey \& Biard, 2012; Kwon et al., 2015; Lisi \& Cavanagh, 2015; Shapiro et al., 2010; Tse \& Hsieh, 2006). This double-drift stimulus (Fig. 1, left) generates extreme misjudgments of the moving target's location which may deviate by as much as several degrees of visual angle from its true location. Surprisingly, this extraordinary perceptual illusion does not affect eye movements to the gabor: immediate saccades to these targets are determined by their physical not their perceived location (Lisi $\&$ Cavanagh, 2015). In contrast, delayed eye movements and pointing go to the perceived position (Lisi \& Cavanagh, 2017; Massendari et al., 2018). These findings suggest that both the true and illusory positions are available in the brain.
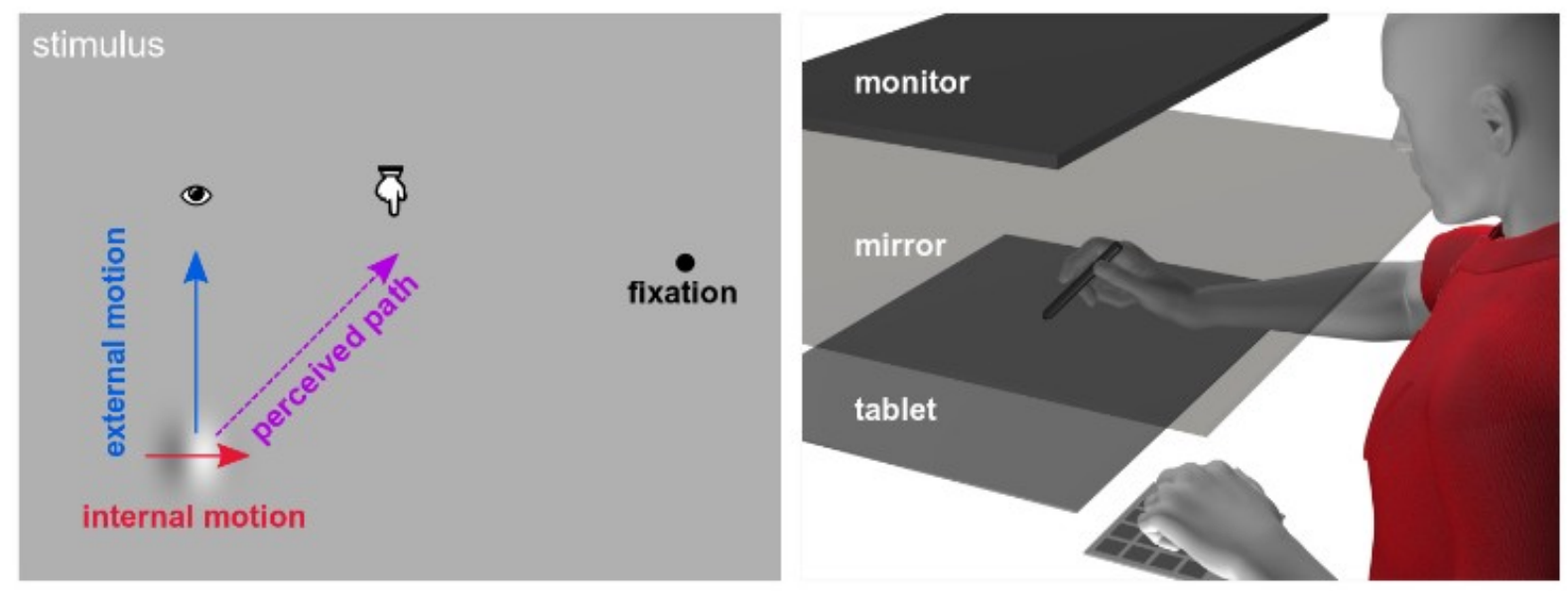

Figure 1: Stimuli and Setup Left: a gabor with internal motion (phase shifts) and external motion, that is viewed peripherally, appears to follow a path deviating from the true path. Right: participants track the perceived trajectory as the gabor moves back and forth along its path.

The strength of the double-drift illusion probably arises because of the very poor location information for a gabor pattern in the periphery when it has the same mean luminance as the background (Cavanagh \& Tse, 2019; Gurnsey \& Biard, 2012; Kwon et al., 2015). With poor positional certainty, the motion information contributes to the location estimate, generating a shift in perceived position. If the gabor itself is not moving (De Valois $\&$ De Valois, 1991), the shift saturates quickly (40 ms: Chung et al., 2007; $100 \mathrm{~ms}$ : Arnold et al., 2007), perhaps because the accumulating evidence of actual location of the stationary gabor patch is sufficient to anchor its position. However, when the gabor itself also moves, as in the double-drift stimulus, there is no stable location information to anchor the position estimate and the build-up continues well beyond $100 \mathrm{~ms}$. The perceived location continues to move away from its physical position by up to several times the size of the gabor. The strength of the illusion, the deviation of perceived path of the gabor from its true path, depends on the speed of both the internal and external motion (Cavanagh \& Tse, 2019; 
Heller et al., 2021; Kwon et al., 2015; Shapiro et al., 2010; Tse \& Hsieh, 2006). In our first experiment, participants track their percept with a stylus as the gabor moves back and forth on its trajectory (Fig. 1) with 5 speeds of the internal motion. We analyze the angle of the tracking to determine if the manual response is susceptible to the illusion.

In our second experiment, we look at the spatial and temporal limits of the illusion. At some point, the illusion stops accumulating. The accumulation can be reset by introducing a temporal break (Lisi \& Cavanagh, 2015) or by distraction attention (Nakayama \& Holcombe, 2020), but it may also reset spontaneously once the accumulation has gone on too long or too far. Informally, some observers have reported that the gabor's path may saturate so that it remains parallel to, but offset from the true path. Others report that the position moves back toward its physical location, either slowly or suddenly, whereupon accumulation begins again. The ability to record trajectories of the perceived position will then allow us to study spontaneous reset outcomes.

\section{Experiment 1: Online tracking}

In this experiment, participants were asked to use a stylus on a drawing tablet to track a moving gabor with some amount of internal motion that elicited the double-drift illusion. We test if tracking the illusion continuously is a good measure of illusion strength, or if it prevents or decreases the illusion.

\section{Methods}

\section{Participants}

For this experiment, 4 participants were recruited from the lab ( 3 female; ages 21 - 31 , mean: 25.75). All participants reported being right handed and had either normal or corrected to normal vision. Procedures were approved by York's Human Participants Review Committee, and all participants provided prior, written, informed consent.

\section{Setup}

Participants used a stylus on a horizontal drawing tablet (Wacom Intuos Pro) to indicate where they perceived the location of a gabor (see Fig. 1, right), as well as a keypad for additional responses. Stimuli were displayed on a downward facing LED monitor (30 Hz, 20", 1680x1050), parallel with the drawing tablet. The stimuli were observed via an upward facing mirror placed exactly between the tablet and monitor, so that the stimuli appear to be in the same horizontal plane as the hand, and the tip of the stylus. Experiments were run in Python 2.7 with PsychoPy (Peirce et al., 2019).

For reporting degrees of visual angle that stimuli spanned ( $d v a$ ), we assumed the participants to be close to the setup, with their eyes at the height of the monitor. While there was no requirement to use the forehead rest, this is a reasonable assumption, since stimuli are only visible when relatively close to the setup. Nevertheless, reported $d v a$ 's are estimates of the size of stimuli. 


\section{Stimuli}

The stimuli were phase-changing gabor patches with a gaussian mask with a sigma (sd) of $0.43 \mathrm{~cm}$ or $\sim 0.59 \mathrm{dva}$, spatial frequency of $0.58 \mathrm{c} / \mathrm{cm}$ or $\sim 0.42 \mathrm{c} / \mathrm{dva}$, vertical orientation, and contrast of $100 \%$. By simultaneously moving the gabor envelope and drifting its internal sine wave, it becomes a 'double-drift stimulus' where the perceived path deviates from the real path. The gabor changed phase at different rates, called 'internal motion' here, specified in cycles per second ( $c p s$ ). The real displacements of the stimulus are along the $\mathrm{Y}$ axis of the monitor, keeping the $\mathrm{X}$ coordinate constant at the middle of the screen, so that a stimulus without internal motion would appear to move toward and away from the participant on the horizontal display.

The motion of the envelope was restricted to the central $13.5 \mathrm{~cm}(\sim 14.0 \mathrm{dva})$ and was set so that the stimulus would move from one end to the other of its path ( a motion we call a 'pass') in $2 \mathrm{~s}$, corresponding to a speed of $\sim 6.75 \mathrm{~cm} / \mathrm{s}$ ( or roughly $7.0 \mathrm{dva} / \mathrm{s}$ ).

\section{Procedure}

In this experiment, participants used their unseen hand and stylus to do continuous online tracking of the double-drift stimulus that moved back and forth along the Y-axis of the horizontal screen for $12 \mathrm{~s}$. Before trial onset, the participant moved the stylus to the middle of the screen, and were given $1.5 s$ to fixate a point on the left or right of the screen. The gabor would then appear at the middle of the screen and start moving. Internal motion could be 3, 1, 0, - 1 or - 3 cps. Since each moving gabor was shown for $12 s$, and it moved from one end to another in $2 \mathrm{~s}$, it completed 5 full and 2 half passes of the path. The internal motion of the gabor was inverted at the far and close ends of the physical path, where external motion was also inverted.

\section{Analyses}

Because there is no set reference point to gauge movement direction against after each direction change, we use instantaneous heading along the trajectory instead. We first segmented the tracking trajectories according to stimulus passes (in between direction changes of the gabor), and removed the noisy tracking during the first and last half second, leaving $1 s$ trajectory segments. We then calculated the instantaneous heading (direction, disregarding velocity or distance) of a smooth spline interpolated version of each trajectory segment (using 101 spline interpolated position samples, to get 100 heading samples). This heading measure should depend on the illusion strength.

We compare the illusory direction of the heading against the predictions of a simple vector combination model of perceived direction from Tse and Hsieh (2006), Cavanagh and Tse (2019) and Heller et al. (2021). In this model, the perceived direction is a simple vector combination of the external $\left(V_{e}\right)$ and internal $\left(V_{i}\right)$ motions and a constant $K$. That is, the deviation from the physical direction is given by: 
If $K$ is 0 , then there is no illusion and the perceived direction matches the external motion direction. However, if $K$ is 1 , then the internal motion has equal contribution with the external motion in determining the perceived direction. For comparison with other work, we will find the value of $K$ that best describes the strength of the illusion in each of our two experiments.

All analyses were done in R 3.6.1 ( $\mathrm{R}$ Core Team, 2019). All data, as well as scripts for the experiments and analyses are available on OSF: https://osf.io/72ndu/.

\section{Results}

In Figure 2 (top right), we plot the average instantaneous heading during the middle second of each $2 s$ pass for each of the 5 internal motion speeds. The average angle of the tracked path is given in degrees deviation from straight forward. It seems that continuous tracking is sensitive to the strength of the illusion, and this is confirmed by a repeated-measures Ftest on the average angles, using internal motion as a within-subjects factor ( $\left.F(4,12)=102.6, p<.001, \eta^{2}=0.97\right)$. With FDR-corrected, paired t-tests, we find a difference in heading between internal motion speeds of 0 cps and both 1 cps $(p=.022)$ and -1 cps ( $p=.025$ ) as well as between -3 and -1 cps and 3 and 1 cps (both $p<.001$ ). This makes it clear that continuous tracking does not prevent the illusion from occurring. We will use the heading angle as a measure of the strength of the illusion.
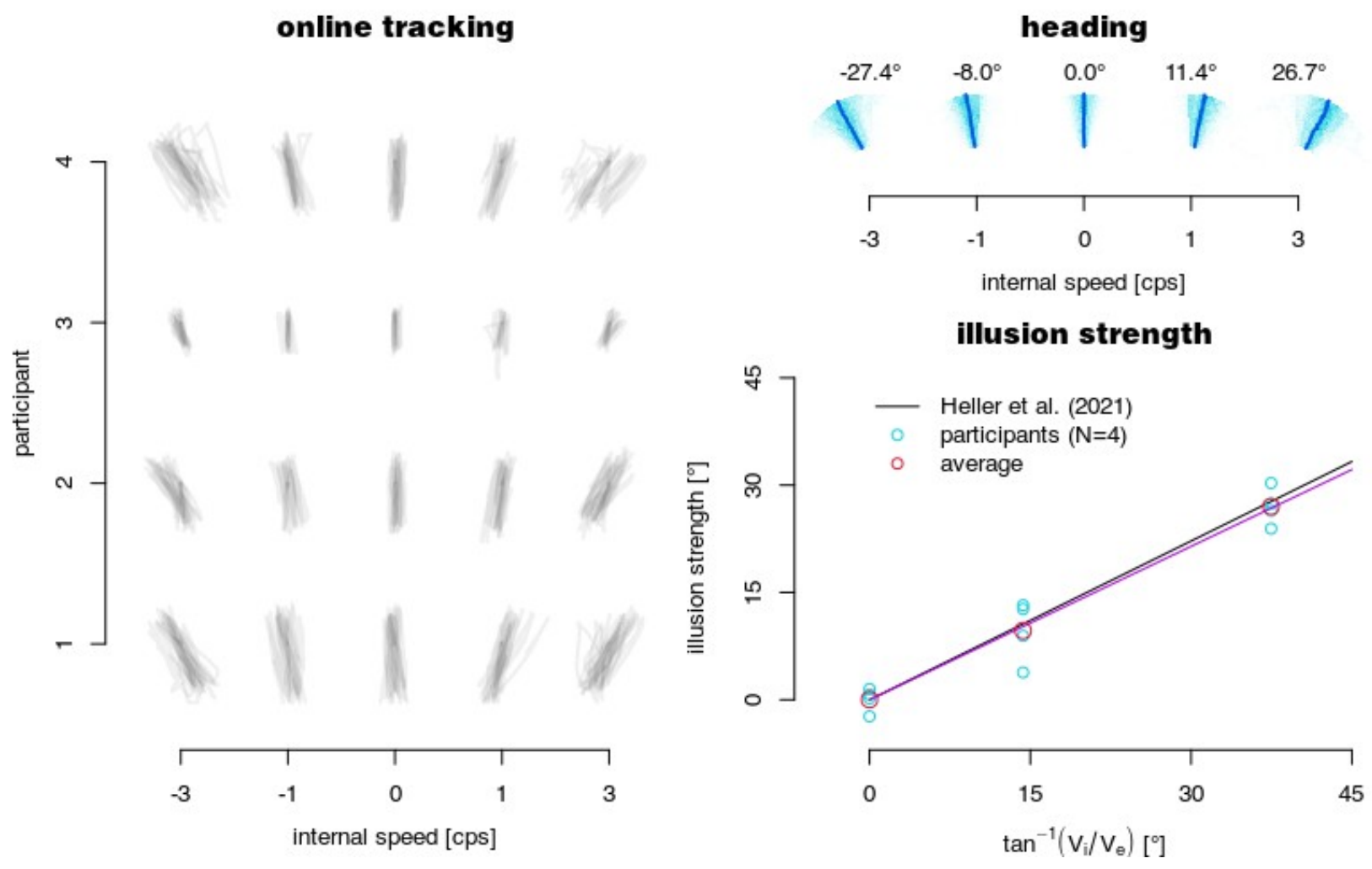

Figure 2: Online Tracking Data. Left: Individual trajectories. In general, the traced paths showed rough linearity. Top right: Average heading of the middle $1 \mathrm{~s}$ of each pass. Distance from origin is time, angle is the heading angle. Dark blue lines the average heading, in light 
blue a polar heatmap with the distribution of heading. Bottom right: The strength of the illusion in degrees angle deviation from the physical path. In red are the average illusion strengths for 0 cps, \pm 1 cps and \pm 3 cps, and in light blue the data for the 4 individual participants. Black line: prediction of the vector combination model of Eq. 1 with $K=0.74$ as in Heller et al. (2021). Purple: best fit to our data, $K=0.72$.

\section{Illusion strength}

The data clearly show the illusion, but does tracking decrease the strength of the illusion compared to simply observing it? In Figure 2 (bottom right), we compare mean illusion strength to that reported previously (Heller et al., 2021). As can be seen the averages from our data here (red circles, purple line) coincide fairly well with the illusion strength predicted by the model fitted to all their data (Eq. 1, black line). That is; tracking the illusion while observing it does not change the strength of the illusion.

\section{Experiment 2: Delayed retracing}

The illusion cannot drift continuously away from the physical path forever. It can be disrupted by a temporal break (Lisi \& Cavanagh, 2015) or by distracting attention (Nakayama \& Holcombe, 2020). These disruptions may cause the perceived path to stay at a fixed offset, travelling parallel to the physical path, or it may return toward the veridical position before resuming the illusory direction (Fig. 3 ). These resets may also occur spontaneously once the accumulation has gone on too long or too far. The purpose of this experiment is to capture this spontaneous resetting and determine its source.

In the previous experiment with online tracking, the duration of each pass in the online tracking experiment was 2 seconds and the manual traces (Fig. 2, left) mostly showed a linear trajectory without saturation or reset of the types shown in Figure 3 (left). The continuation of the accumulation may be a result of the active tracking - either the extra attention required to track increased the extent of the accumulation or possibly the temporal demands of the tracking caused the participants to average their traces over any breaks. To address this, we switched to a delayed, offline recording of the perceived path. 

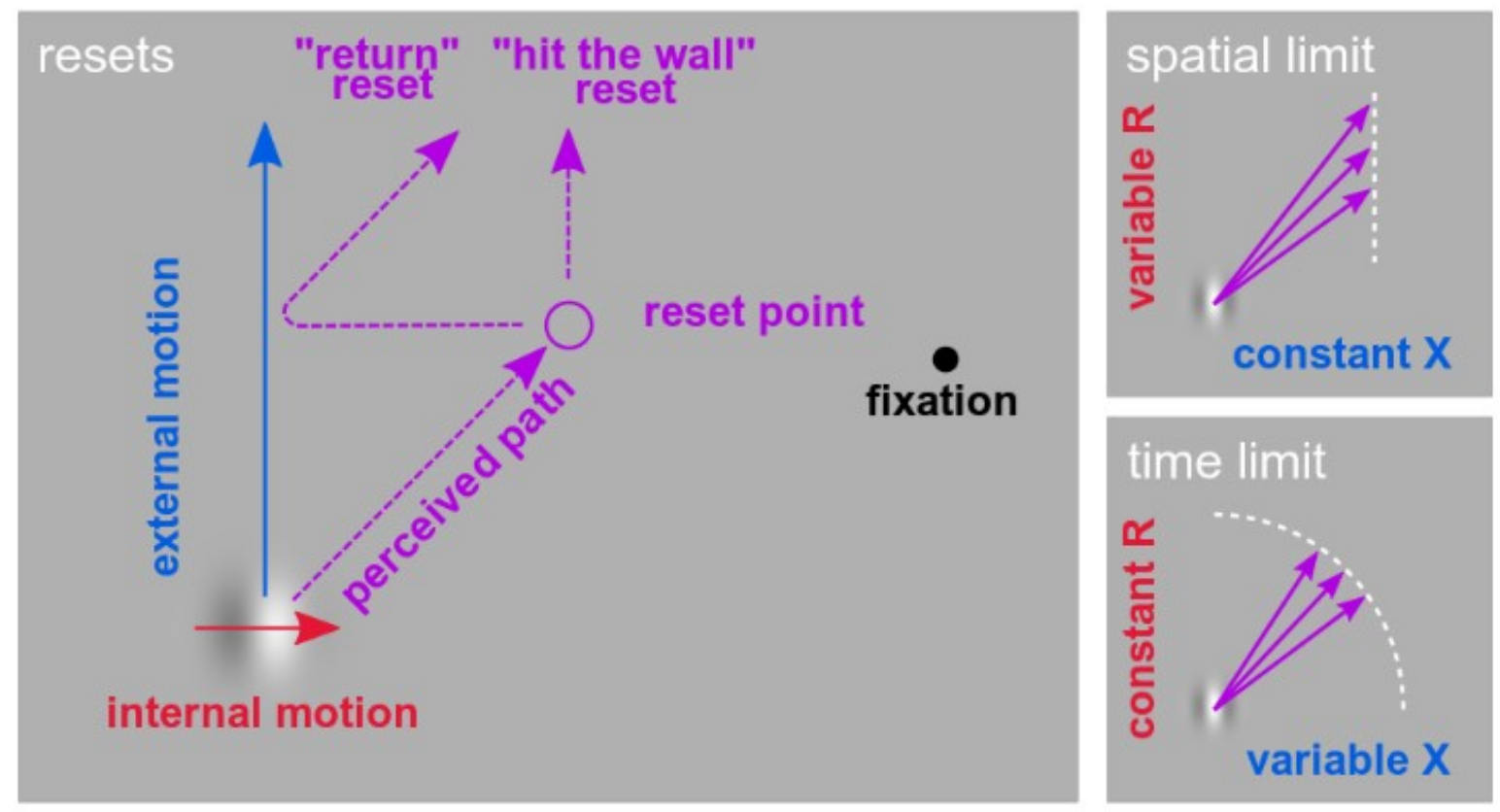

Figure 3: Double-Drift Resets. Left: At some point, the perceived position may stop moving further away from its actual path. For some people this takes the form of a 'return' reset, back toward the true position, for others it means the illusory position remains at a fixed offset moving parallel to the real path, or some combination of the two. Top right: if these resets are due to a spatial limit on the size of the illusory position shift away from the true vertical path, the locations of the resets should have a constant $X$ coordinatee, the spatial offset from the vertical path. Bottom right: if they occur instead after some period of time, resets should have a constant $Y$ coordinate, the time since the gabor's motion began.

We were interested, in particular, in whether the limitation of the accumulation would be set by space or time. Since both the true and the illusory position appear to be available in the visual system (the true position drives saccades whereas the illusory position drives perception, Lisi \& Cavanagh, (2015), it is possible that resets occur once the distance between the real and perceived positions exceeds some limit. We can speculate that this spatial limit would be related to the positional uncertainty of the gabor. When the position information is reliable, the resets would occur with very little illusory deviation, keeping the perceived path close to the true path. When the positional uncertainty is high, in the periphery, with the gabor's mean luminance matched to the background (Cavanagh $\&$ Tse, 2019; Gurnsey $\&$ Biard, 2012), the spatial offset could be quite large before exceeding the range of positional uncertainty around the gabor's true position. Under this hypothesis, the timing of spontaneous resets will depend on the spatial offset from the true path which will be directly proportional to the internal speed (for a constant external motion). On the other hand, if spontaneous resets are the result of the temporal limitations of the integration process, resets would occur after a certain amount of time, independently of the internal speed and the spatial offset it creates. Investigating the time and location of resets in the recorded trajectories, allows us to distinguish between a spatial limit to the illusion and a 
temporal limit. This in turn will inform us of the processes underlying the perception of the position of moving objects.

To do so, we ask participants to re-trace the perceived path of a double-drift stimulus, immediately after viewing the stimulus, a method used by Nakayama \& Holcombe (2020). This allows participants more time to carefully reproduce the path. We use the recorded trajectories to assess points where resets occurred, whether they were a discontinuity in orientation reflecting a saturation (hit the wall reset, Fig. 3) or a discontinuity in position reflecting a jump back to the real location. These reset points can then be used to determine if resets are time-limited or space-limited.

\section{Methods}

\section{Participants}

For this experiment, 9 participants were recruited from the lab (6 female; ages 19 - 27, mean: 22.8). All participants reported to be right handed and had either normal or corrected to normal vision. Procedures were approved by York's Human Participants Review Committee, and all participants provided prior, written, informed consent.

\section{Setup and Stimuli}

The setup was the same as used in the online tracking experiment, except that we now also used a small keypad, for responses (Fig. 1, right). Stimuli were identical to those used in the online tracking experiment, but now always started at the near end of the workspace and moved away from the participants. The double drift stimuli would use either 3 or 4 seconds to move $13.5 \mathrm{~cm}$, corresponding to speeds of $\sim 4.5$ and $\sim 3.75 \mathrm{~cm} / \mathrm{s}$ or $\sim 4.7$ or $\sim 3.5 \mathrm{dva} / \mathrm{s}$, and had an internal drift of 2, 3 or 4 cps .

\section{Procedure}

In experiment 2, participants first watched a double-drift stimulus and then either reproduced the perceived path of the stimulus by retracing it on the tablet, or indicated the initial movement direction by changing the orientation of a line originating in the same position as the doube-drift stimulus (data not used, but corresponds to illusion strength determined from trajectories). More details are given below. This provided more time for the participants to reproduce any resets, i.e. without the need for real-time tracking. The retracing task was done in half of 8 blocks (the other half of the blocks are not used). Block types were alternated, and the order was counterbalanced across participants. Each block used the 6 combinations of two external speeds ( corresponding to 3 or $4 \mathrm{~s}$ presentation time ), and three internal speeds ( 2, 3 or 4 cps ), 6 times, for a total of 36 trials per block. Each of the combinations of internal and external speed was presented 24 times in total.

In both kinds of trials, participants first had to move the stylus to the start position of the gabor, and then fixate a point to the left or right of the centre of the workspace. The doubledrift gabor started at the near end of the display, and moved along the horizontal display away from the participant, always making a single, forward pass. After the gabor had disappeared, participants could respond in one of two ways. When re-tracing the perceived 
path with the stylus on the tablet, the drawn path would show up as a red line. Participants could "key in" their response by pressing ENTER, or start over by pressing ESCAPE. There was no time-limit for the response. Re-tracing the perceived paths should allow capturing spontaneous resets of the illusion, if there are any.

The individual trajectories reveal some points that are potential spontaneous resets that we try to extract. Using a smooth spline interpolated version of the trajectory we detect rightleft direction changes, based on sign changes. Since the initial movement is often jittery, we only take direction changes further than $\sim 0.39 \mathrm{~cm}(\sim 0.23 \mathrm{dva})$ away from the starting point. The first (if any) of the remaining direction changes, are then taken as reset points. To keep the horizontal and vertical (straight ahead) coordinates of the reset points isometric they are given in centimeters. The distance from origin can then be converted to time as $1 \mathrm{~cm}=3.375 \mathrm{~s}$ in the $4 s$ condition and $1 \mathrm{~cm}=4.5 \mathrm{~s}$ in the $3 \mathrm{~s}$ condition. Participants' ability to retrace perceived trajectories might vary, but normalizing trajectories so that the final Y coordinate is at $13.5 \mathrm{~cm}$ does not noticably change the results. sample halfway between the starting point and the detected reset point. We then use the angular difference between the gabor's real trajectory and a straight line drawn through that point and the start of the trajectory as a measure of illusion strength.

\section{Results}

In Figure 4 (middle), we show the trajectories for participant 4 in the condition with 3 cps internal motion and $4 s$ passes. This particular participant's re-traced paths show clear 'return' resets, all of which are detected by our simple algorithm. Other participants' data are less clear. 

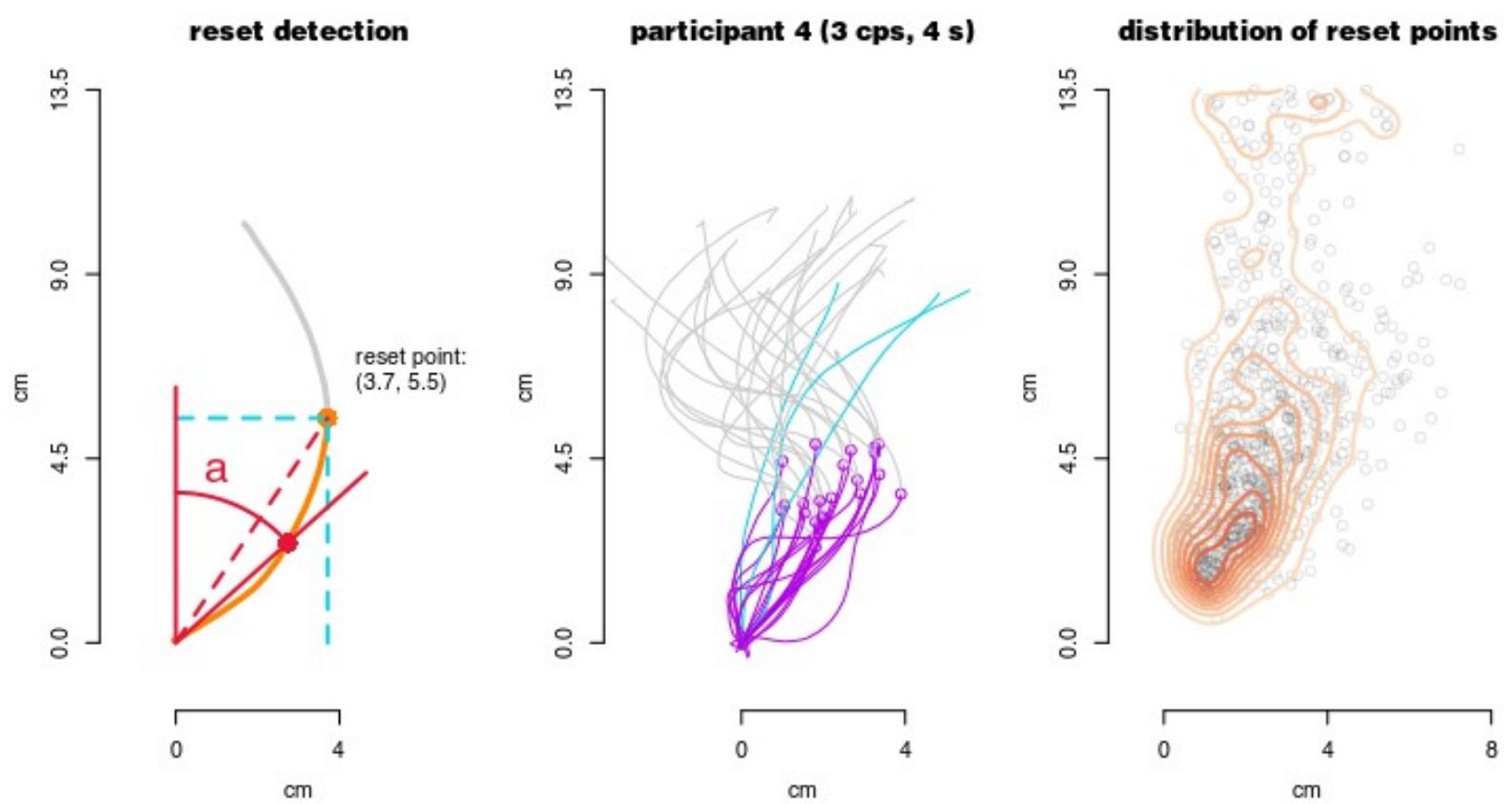

Figure 4: Re-tracing Data. Left: Reset points are taken at the first sample in a splinedsmoothed re-traced trajectory that goes in the direction opposite that of the expected illusion. Orange and gray lines: example trajectory. Orange dot: reset point. Red dot: point at half the distance to the reset point. Angle a: measure of illusion strength. Middle: One participant's trajectories with very clear 'return' resets at the purple dots. (Purple lines: trajectories up to resets, gray lines: trajectories after resets, blue lines: trajectories that had no resets in the retracing.) Other participants' data were not this clear. Right: Distribution of all 859 detected reset points.

On average, this method detects resets in $\sim 75 \%$ of trials. For three participants, only about half the trials showed resets $(42 \%, 44 \%$ and $58 \%)$. For the remaining six participants the algorithm detected resets in $72 \%-99 \%$ of trials, with an average of $88 \%$. The minimum number of resets in each of the six conditions for any participant is 7 (out of 24 trials), with an average of $\sim 17$. Since participants experiencing a "hit-the-wall reset" (Fig. 3) as opposed to a "jump" reset, might not always display a detectable direction change in their paths, $50 \%$ of trials with resets seems reasonable. Either type of perceived resets appears valid, and the average reset positions still indicates some limit to the illusion. Therefore, we kept all participants and resets in our analysis (reset points in Fig. 4, right).

As before, we compare the strength of the illusion (see Fig 5, left), with an earlier study (Heller et al., 2021). A line through the origin with slope 0.51 ( $K$ from Eq. 1 ) predicts illusion strength fairly well in these data, which is weaker than the $K=0.74$ found by Heller et al. (2021) and lower than the $K=0.72$ found in Experiment 1 with online tracking. While the illusion is less strong, it is nevertheless there and depends on the relationship between internal and external speed as in previous studies. 


\section{Limits on the Double-Drift IIlusion}

307

308

Now we look at the distribution of reset points, and whether they can be explained by a temporal or spatial limit. In Figure 4 (middle), we can see that the reset points vary more along the $y$-axis, than along the $\mathrm{x}$-axis. That is, it looks like predicting reset points from a single $\mathrm{X}$ coordinate (or spatial limit) than predicing reset points from a given time. However, there is also considerable variance, so that fitting any line through the data, will necessarily leave errors.

illusion strength

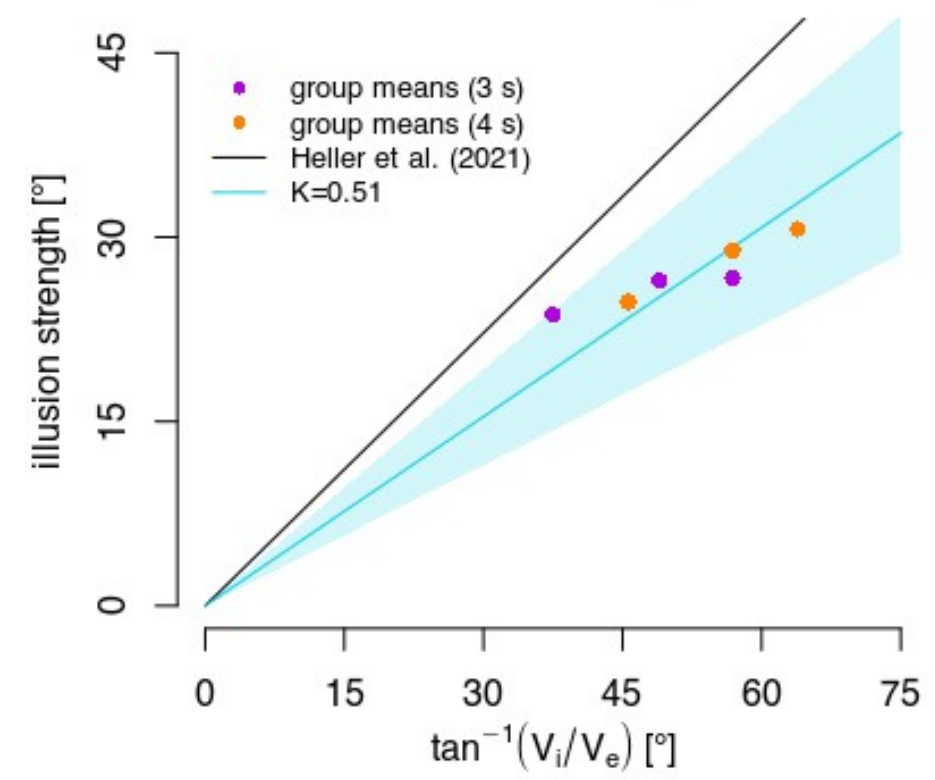

model reset limits

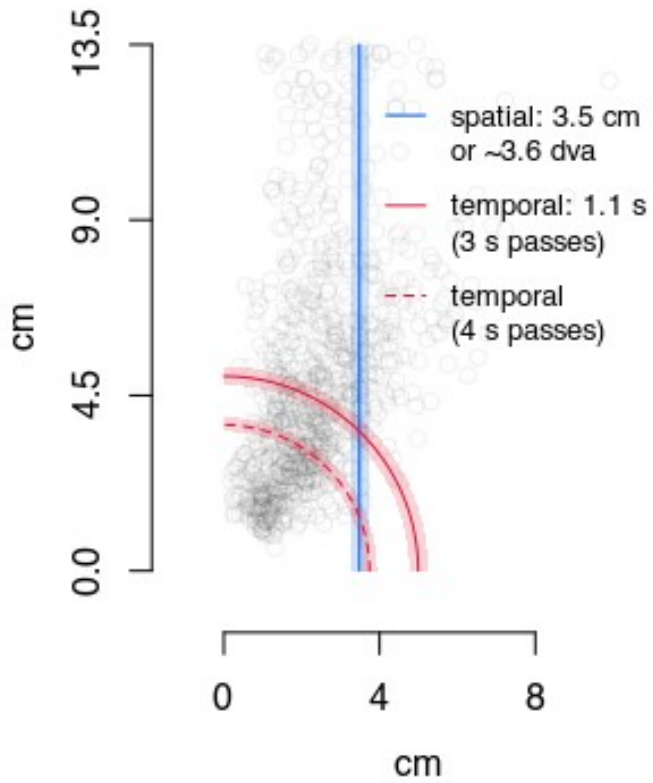

Figure 5: Illusion strength and reset models. Left: Illusion strength as in Figure 2 (bottom right); plotted against predicted strength from Eq.1: the arctangent of the ratio of internal to external velocities. Purple dots are the group means from $3 s$ passes, orange from $4 s$ passes (2, 3 and 4 cps internal speeds). Predictions from the vector combination model in Eq. 1: black line; $K=0.74$ as in Heller et al. (2021), light blue line; best fit here at $K=0.51$ and the shaded area shows a 95\% confidence interval. Right: Model limits shown here come from a weighted bootstrapping across participants, lines are median parameter values, shaded areas the 95\% confidence intervals of the limits. Gray dots are the reset points from all participants. While a spatial limit provides the better fit, neither a spatial nor a temporal limit explains the data well.

We test this with a series of simple models. First, for every reset point (with coordinates $X$ and $Y$ ) we use the illusion strength based on a point earlier in the trajectory, expressed as either an angle (Fig. 4, left: $a=\arctan (X / Y))$ or a slope $(s=\sin (a) / \cos (a))$ to predict reset points.

If the perceived path of the gabor is approximated by a straight line (up to a reset), and reset points occur at a set limit, then a spatial limit $\left(L_{x}\right)$ would equal the $\mathrm{x}$-coordinate and predict the $y$-coordinate, $y$, of the first reset: 
And a temporal limit $\left(L_{t}\right)$ would predict both coordinates of the reset, $x$ and $y$ :

Where $L_{t}$ and $L_{x}$ are a temporal and spatial limit (in seconds and cm respectively) that are free parameters, $a$ and $s$ correspond to the calculated angle and slope expressing the strength of the illusion, and speed $(v$ in $\mathrm{cm} / \mathrm{s}$ ) is given by the stimulus. If we fit the two simple models for each participant separately, by minimizing the mean square error (in isometric space, with absolute distance in centimeters between predicted and actual reset points), most participants are best fit with a spatial limit (MSE: 6/9, AIC: 7/9). This indicates that in this data, a spatial limit explains the reset points best, but neither limit explains the data very well.

To obtain population level values for the spatial and temporal limits, we resample reset points from each participant, using the smallest number of detected reset points that fall in the first quadrant, in the group (44), to ensure that each participant contributes the same number of reset points. Both models based on a spatial limit (eq. 2) and on a temporal limit (eq. 3) were fit 1000 times (Fig. 5, right). The median fits use a spatial limit of $3.5 \mathrm{~cm}$ or $\sim 3.6 d v a$ and a temporal limit of $1.1 \mathrm{~s}$. We explored other modeling approaches (e.g. based on various distributions of likelihoods or combining limits) but these show the same pattern of results: spatial limits are somewhat favoured, but temporal limits can not be excluded (see OSF repository: https://osf.io/72ndu/).

\section{Discussion}

We first tested whether manual tracking of a double-drift stimulus is susceptible to the illusion as reported by perceptual judgments, pointing (Lisi \& Cavanagh, 2017), and memory saccades (Massendari et al., 2018). The results of this online tracking experiment show a close agreement between the angle of the manual tracking and the illusion strength expected based on the model from earlier perceptual measures (Cavanagh $\&$ Tse, 2019). However, since no unambiguous resets were captured in the traces of this experiment, we followed up with a second experiment that did record evidence of resets.

To measure resets, we asked participants to draw the perceived path of the stimulus after a single pass. The orientation of the drawn traces reflect the strength of the illusion, and in most trials there was a clear reset point, for either a "return" reset or a "hit-the-wall" reset. We then set out to test if these spontaneous resets are triggered by a limiting distance of drift away from the physical location, or by a specific limit of time. The results showed that the resets are slightly better explained by the distance from the true position, but a temporal limit is not ruled out.

Recently, Kwon, Tadin, and Knill (2015) proposed a Bayesian object tracking model to explain the double-drift illusion. In their model, when the position signals are weak, the 
motion signals bias perceived position away from the true location. In their case, this deviation saturated after about $200 \mathrm{~ms}$ and the perceived path then continued parallel to the physical path, as in the "hit the wall" resets we described above (Kwon et al., 2015, Fig. 3 ). In contrast to their model, our participants in Experiment 2 showed quite variable timing for the reset point where the accumulation of offset ended. The resets were more stable in terms of spatial offset (Fig. 4, middle) but much more variable in terms of time from the start of the gabor's motion. In any case, the temporal limit of these reset points was not $200 \mathrm{~ms}$ but $1.1 s$ and fell into a wide range from $0.5 s$ to almost $4 s$ across trials (see Fig. 4). Moreover, 4/9 of our participants regularly reported "return" resets that the Kwon et al. (2015) model does not predict. The "return" resets suggest that both the physical and perceived locations are available to the visual system and that when the deviation exceeds some limit, the perceived location returns toward the physical location. At that point, the build-up of the illusion may start again.

Recently, it has been suggested that resets can be triggered by a distraction of attention (Nakayama \& Holcombe, 2020). The participants in this experiment also retraced the perceived path and reported "return" resets. Nakayama and Holcombe also suggest that planned eye movements can trigger a "return" reset back to the physical path. In our experiment, we did not control for eye-movements, so we can't directly investigate whether eye movements accompanied the "return" resets that participants reported. However, the modestly better performance of the spatial limit model here suggests that spontaneous resets are not randomly distributed - as they might be if eye-movements or shifts of attention are the only way to elicit resets.

We find that manual tracking of the double-drift stimulus during its motion did show the expected illusion. Few if any resets were detected during the online tracking, either because of the additional attentional requirements or because of the short duration (2 s). Participants did report spontaneous resets in delayed, single-pass re-tracing of Experiment 2. The resets were best explained by a limiting spatial offset from the true stimulus location, but do not exclude a temporal limit. These findings may either indicate there are multiple causes for spontaneous resets or that a re-tracing method is not suitable to resolve what causes this phenomenon. Either way, both some amount of time from stimulus onset until reset and too large a conflict between retinal and perceived position leading to resets remain possible.

\section{Acknowledgements}

The research was support by funding from NSERC Canada (PC and DYPH), as well as by the Department of Psychological and Brain Science, Dartmouth College (PC).

\section{References}

Arnold, D., Thompson, M., \& Johnston, A. (2007). Motion and position coding. Vision

Research, 47(18), 2403-2410. https://doi.org/10.1016/j.visres.2007.04.025 
Cavanagh, P., \& Anstis, S. (2013). The flash grab effect. Vision Research, 91, 8-20. https://doi.org/10.1016/j.visres.2013.07.007

Cavanagh, P., \& Tse, P. U. (2019). The vector combination underlying the double-drift illusion is based on motion in world coordinates: Evidence from smooth pursuit. Journal of Vision, 19(14), 2. https://doi.org/10.1167/19.14.2

Chung, S. T. L., Patel, S. S., Bedell, H. E., \& Yilmaz, O. (2007). Spatial and temporal properties of the illusory motion-induced position shift for drifting stimuli. Vision Research, 47(2), 231-243. https://doi.org/10.1016/j.visres.2006.10.008

De Valois, R. L., \& De Valois, K. K. (1991). Vernier acuity with stationary moving gabors. Vision Research, 31(9), 1618-1626. https://doi.org/10.1016/0042-6989(91)90138-U

Duhamel, J.-R., Colby, C. L., \& Goldberg, M. E. (1992). The updating of the representation of visual space in parietal cortex by intended eye movements. Science, 255(5040), 90-92. https://doi.org/10.1126/science.1553535

Gurnsey, R., \& Biard, M. (2012). Eccentricity dependence of the curveball illusion. Canadian Journal of Experimental Psychology, 66(2), 144-152. https://doi.org/10.1037/a0026989

Heller, N. H., Patel, N., Faustin, V. M., Cavanagh, P., \& Tse, P. U. (2021). Effects of internal and external velocity on the perceived direction of the double-drift illusion. Journal of Vision, 21(8). https://doi.org/10.1167/jov.21.8.2

Hogendoorn, H. (2020). Motion Extrapolation in Visual Processing: Lessons from 25 Years of Flash-Lag Debate. Journal of Neuroscience, 40(30), 5698-5705. https://doi.org/10.1523/JNEUROSCI.0275-20.2020

Kwon, O. S., Tadin, D., \& Knill, D. C. (2015). Unifying account of visual motion and position perception. Proceedings of the National Academy of Sciences of the United States of America, 112(26), 8142-8147. https://doi.org/10.1073/pnas.1500361112

Lisi, M., \& Cavanagh, P. (2015). Dissociation between the perceptual and saccadic localization of moving objects. Current Biology, 25(19), 2535-2540. https://doi.org/10.1016/j.cub.2015.08.021

Lisi, M., \& Cavanagh, P. (2017). Differential spatial representations guide eye and hand movements. Journal of Vision, 17(12). https://doi.org/10.1167/17.2.12

Massendari, D., Lisi, M., Collins, T., \& Cavanagh, P. (2018). Memory-guided saccades show effect of a perceptual illusion whereas visually guided saccades do not. Journal of Neurophysiology, 119(1), 62-72. https://doi.org/10.1152/jn.00229.2017

Nakayama, R., \& Holcombe, A. O. (2020). Attention updates the perceived position of moving objects. Journal of Vision, 20(21). https://doi.org/10.1167/jov.20.4.21

Nijhawan, R. (1994). Motion extrapolation in catching. Nature, 370(6487), 256-257. https://doi.org/10.1038/370256b0 
443 Peirce, J. W., Gray, J. R., Simpson, S., MacAskill, M. R., Höchenberger, R., Sogo, H., Kastman, E.,

$444 \quad \mathcal{E}$ Lindeløv, J. (2019). PsychoPy2: Experiments in behavior made easy. Behavior Research

445 Methods, 51(1), 195-203. https://doi.org/10.3758/s13428-018-01193-y

446 R Core Team. (2019). R: A language and environment for statistical computing. R Foundation

447 for Statistical Computing. https://www.R-project.org/

448 Shapiro, A., Lu, Z.-L., Huang, C.-B., Knight, E., \& Ennis, R. (2010). Transitions between central 449 and peripheral vision create spatial/temporal distortions: A hypothesis concerning the

$450 \quad$ perceived break of the curveball. PLoS ONE, 5(10), e13296.

451 https://doi.org/10.1371/journal.pone.0013296

452 Tse, P. U., \& Hsieh, P. J. J. (2006). The infinite regress illusion reveals faulty integration of

$453 \quad$ local and global motion signals. Vision Research, 46(22), 3881-3885.

454 https://doi.org/10.1016/j.visres.2006.06.010 